\title{
Vitamin D deficiency and its characteristics among patients with acute stroke at a national referral hospital in Kampala Uganda
}

\author{
Daniel S. Kiggundu ${ }^{1 *}$, Edrisa Mutebi ${ }^{1}$, Davis Kibirige ${ }^{2}$, Rebecca Boxer ${ }^{3}$, Barbara Kakande $^{4}$, Brian K. Kigozi ${ }^{5}$
} and Elly Katabira'

\begin{abstract}
Background: Vitamin D deficiency has been associated with acute stroke and other cardiovascular diseases in the developed world. Low 25-hydroxyvitamin D (25OHD) has been described in some populations in Sub-Saharan Africa (SSA) in spite of adequate sunshine all year round. There is no information on the magnitude of vitamin D deficiency among patients with stroke and other cardiovascular diseases in Uganda or SSA. The aim of this study was to determine the burden and characteristics of vitamin D deficiency among patients with acute stroke, the most common form of cardiovascular events in SSA.

Methods: We conducted a cross-sectional study between October 2012 and March 2013. We consecutively recruited 142 subjects with acute stroke admitted to the medical wards of Mulago hospital. We administered a pre-tested questionnaire to the study participants, and did a detailed physical examination and laboratory evaluation. Serum levels of 250HD were determined using an electrochemiluminescence assay. Data were analyzed using STATA version 12 software.

Results: The prevalence of vitamin D deficiency $(25 \mathrm{OHD}<20 \mathrm{ng} / \mathrm{ml})$ was $15 \%$. Longer hours of sunshine exposure decreased the likelihood vitamin D deficiency significantly (adjusted OR 0.85, $p=0.03$ ). Higher HDL cholesterol had a significant inverse association with vitamin D deficiency (adjusted OR 0.15, $p=0.02$ ). In addition, the likelihood of vitamin D deficiency increased with rising age (adjusted OR 1.03, $p=0.05$ ).

Conclusions: There was a relatively low burden of vitamin $D$ deficiency among patients with acute stroke in Uganda. With increasing longevity and indoor lifestyles vitamin $D$ deficiency may assume a greater role in stroke and other cardiovascular diseases in tropical sub Saharan Africa. Future studies on the mechanisms of vitamin $\mathrm{D}$ deficiency and its relationship to outcomes among patients with stroke may be necessary.
\end{abstract}

Keywords: Vitamin D deficiency, Stroke, Uganda

\section{Background}

Vitamin D deficiency is a widespread health problem, affecting up to 1 billion people worldwide [1]. It has gained increasing recognition in the developing world, including Sub- Saharan Africa (SSA) where sunshine prevails all year round [2-4]. Previous studies in SSA have found widely varying prevalence of vitamin D deficiency in a tropical region with good quality UVB radiation

\footnotetext{
* Correspondence: dchiggundu@gmail.com

'Department of Medicine, College of Health Sciences, Makerere University, Kampala, Uganda

Full list of author information is available at the end of the article
}

[5-8]. For example, studies in Uganda which straddles the equator have reported a prevalence of vitamin $\mathrm{D}$ deficiency ranging between 9-20\% among healthy individuals $[5,6]$. In central Ethiopia $\left(8^{0} 33 \mathrm{~N}\right.$ to $\left.8^{0} 36 \mathrm{~N}\right)$ a prevalence of up to $61 \%$ has been reported among otherwise healthy urban schoolchildren [8]. Latitude, season, cultural norms, religious practices, low awareness, low knowledge/health literacy, indoor lifestyles, urban living, skin pigmentation, malnutrition, diet, co-morbidities like tuberculosis, and drugs may contribute to vitamin D deficiency in the SSA region $[2,3,9,10]$. 
Classically, vitamin D deficiency is known for its effects on bone, causing reduced mineralization, rickets and osteomalacia $[1,3]$. However, many new roles of vitamin D have recently come to light, with more consequences attributed to its deficiency [1]. Vitamin D deficiency is increasingly associated with infectious diseases like tuberculosis and severe malaria $[6,11,12]$, and non communicable diseases such as cancer, depression, dementia, multiple sclerosis, cardiovascular diseases, and stroke $[1,6,13-16]$.

Cardiovascular diseases are on the rise in SSA, where they are projected to become the leading cause of morbidity by 2030 [17]. Stroke contributes the biggest proportion of cardiovascular disease in SSA and it is responsible for up to $3 \%$ of all adult deaths $[17,18]$.

Studies in non-tropical settings have described higher burden of vitamin D deficiency among acute stroke patients than non- stroke individuals in the same environment $[16,19]$. Suboptimal vitamin $D$ has been associated with many modifiable cardiovascular risk factors, including physical inactivity, hypertension, dyslipidemia, obesity and diabetes mellitus, including among migrant populations from Sub Saharan Africa [13, 15, 20-23]. Furthermore, longitudinal studies have demonstrated that vitamin $\mathrm{D}$ is an independent predictor of cardiovascular disease and stroke $[13,19]$. The relationship of vitamin $\mathrm{D}$ deficiency to large vessel stroke and other atherosclerotic cardiovascular diseases may be mediated by atherogenic proinflammatory cytokines that foster atherosclerotic vascular changes and plaque instability $[24,25]$.

Among stroke patients, vitamin D deficiency has been shown to predict severity and adverse outcomes including death $[19,26]$. Stroke survivors with vitamin D deficiency may be prone to bone loss and pathological fractures [16, 26]. Supplementation with oral 25OHD has been suggested as a potential strategy to reduce cardiovascular diseases and other non-communicable diseases [27]. Ongoing large scale clinical studies in North America are evaluating the role of $25 \mathrm{OHD}$ replacement in the prevention of stroke among other non communicable diseases [28].

Currently, there are few data on the magnitude of vitamin $\mathrm{D}$ deficiency in patients with cardiovascular diseases in tropical SSA with its unique latitude, indigenous peoples and culture. We therefore sought to determine the prevalence and characteristics of vitamin D deficiency in hospitalized patients with acute stroke at Mulago national referral and teaching hospital (Mulago hospital) in Kampala, Uganda.

\section{Methods}

\section{Study design and site}

This was a cross- sectional study conducted on the medical wards of Mulago hospital from October 2012 to
March 2013. The medical wards receive 20-30 stroke patients on the neurology service monthly [29]. Mulago hospital is a 1600 bed-capacity hospital and it is the largest referral hospital in Uganda. Uganda is located on the East Africa plateau and spans the equator from approximately $1^{\circ} \mathrm{S}$ to $4^{\circ} \mathrm{N}$. Kampala city is located near the equator at latitude of $00^{\circ} 20^{\prime}$ North, with constant sunshine all year round and two rainy seasons; one from March to May and the other between September and December.

\section{Study definitions and participants}

For the purposes of this study, acute stroke was defined as a stroke that had lasted not more than 2 weeks from the onset of symptoms. Stroke diagnosis was made as per the classical World Health Organisation definition that identifies stroke as 'a clinical syndrome typified by rapidly developing signs of focal or global disturbance of cerebral functions, lasting more than $24 \mathrm{~h}$ or leading to death, with no apparent causes other than of vascular origin'. Patients were included with or without radiological confirmation using brain computerised tomography (CT) scan.

A period within 2 weeks of symptoms was used to designate acute stroke, in order to reflect pre-stroke concentrations of $25 \mathrm{OHD}$, with minimal post-stroke influence. This was chosen because 25OHD has a half life of 2-3 weeks, depending on 25OHD type and concentration, vitamin $\mathrm{D}$ binding protein genotype and concentration, and race [30].

The participants' 25OHD status was defined basing on the Endocrine Society Clinical Practice Guidelines on evaluation, treatment and prevention of vitamin $\mathrm{D}$ deficiency [31].

Normal 25OHD level was defined as serum $25 \mathrm{OHD} \geq 30 \mathrm{ng} / \mathrm{ml}$. Vitamin D insufficiency was defined as serum $25 \mathrm{OHD} 20-<30 \mathrm{ng} / \mathrm{ml}$. Vitamin D deficiency was used to describe $25 \mathrm{OHD}<20 \mathrm{ng} / \mathrm{ml}$. That included severe vitamin D deficiency with $25 \mathrm{OHD}<10 \mathrm{ng} / \mathrm{ml}$.

During the study period, consecutive sampling was used to approach all adults (aged $\geq 18$ years) admitted to the medical wards with a diagnosis of acute stroke made by the ward team. Consent was obtained from patients or their relatives, and patients who fulfilled the study definition of acute stroke were consecutively enrolled into the study. Patients for whom no history could be obtained were excluded from the study.

\section{Data collection}

After obtaining informed consent, pre-tested questionnaires were administered by the study team to obtain the socio-demographic characteristics, medical history (history of hypertension, diabetes mellitus, HIV), drug history (anti-convulsants, anti-retroviral therapy, multi- 
vitamin supplements, and anti tuberculous drugs), exercise and estimated duration spent outdoors on a typical day. HIV status was determined using a routine testing and counselling algorithm of Mulago Hospital.

Diabetes mellitus (DM) status was decided by obtaining history of a previous diagnosis of diabetes mellitus, or a random glucose measurement $\geq 11.1 \mathrm{mmol} / \mathrm{L}$. History of a previous diagnosis of hypertension was obtained. Blood pressure was measured using the Omron M2 Eco digital blood pressure machine. Where the attending clinical team reported a diagnosis of hypertension based on additional clinical findings, it was accepted as part of the history. However, due to the possibility of reactive changes in blood pressure during acute stroke, blood pressure measurements were not used to define hypertension. Lipid profiles and blood glucose were measured without strict observance of fasting state due to practical challenges in enforcing fasting among often severely ill patients.

Waist circumference was measured to assess abdominal obesity using the International Diabetes Federation (IDF) criteria [32]. The participants' Glasgow Coma Scale (GCS) score and stroke type as identified on brain CT scan were also documented (Additional file 1).

\section{Laboratory assessment \\ Sample collection}

From each participant, a blood sample $(5 \mathrm{ml})$ was drawn aseptically into a plain blood sample tube (BD Vacutainer) and allowed to coagulate at room temperature.

The blood samples were transported to the clinical chemistry laboratory of Mulago hospital within $1 \mathrm{~h}$ of collection, centrifuged and the extracted sera stored at $-20{ }^{\circ} \mathrm{C}$ in a refrigerator until testing.

\section{Diagnostic testing}

Chemistry analysis was done on thawed sera, using the fully automated COBAS 6000 (Roche Diagnostics GmbH) machine. Serum 25OHD concentration was determined using an electrochemilumniscence immunoassay: the Elecsys Vitamin D Total assay (Roche diagnostics, MannheimGermany; Reference number 05894913190 V4). It measures total serum 25OHD concentrations in the range of 3-70 ng/ml. The COBAS 6000 analyzer was also used to measure the serum lipids.

Measurement of albumin, calcium concentration, creatinine and lipid profile was also done. (Lipid profile included low density lipoprotein cholesterol [LDLC] and high density lipoprotein cholesterol [HDLC].

\section{Statistical analysis}

Based on the prevalence of vitamin D deficiency (defined as vitamin D levels $<20 \mathrm{ng} / \mathrm{ml}$ ) of $9 \%$ among healthy individuals in a study in Mulago Hospital, Kampala [5] with a $95 \% \mathrm{CI}$ and desired precision of estimation of
0.05 , a sample size of 126 patients was obtained. We enrolled 142 patients consecutively over a 6 months study period; however, due to logistical constraints we did not adjust sample size for associated factors.

Data were entered using Epi data entry version 3.1 and exported to Stata version 12.1 software, for statistical analysis. Patient characteristics were reported in tables as proportions. Continuous variables were reported as median and inter-quartile range (IQR). Relationship of 25OHD and continuous variables was explored with Pearsons correlation coefficient.

With the study goal to identify characteristics associated with vitamin D deficiency, factors were entered into a logistic regression model. All statistically significant factors from the univariate analysis with $p<0.1$ were entered into the multivariable logistic regression model. In addition, categorical outcomes that were not binary were considered for inclusion if they had a significant overall main effect. Important confounder variables including gender, diabetes mellitus, central obesity and a history of hypertension were also included in the model by a priori decision regardless of statistical significance. A $p$-value of $<0.05$ and confidence intervals not including 1 were considered to be statistically significant at multivariable analysis.

\section{Study approval}

The study was approved by the Makerere University College of Health Sciences, School of Medicine Research and Ethics Committee. For all patients, written informed consent was obtained prior to enrolment in the study from their relatives or care takers. All participants were given information on the positive effect of exposure to sunshine on 25OHD levels. Where vitamin D deficiency was found, the result was explained to the patient/caretaker by telephone, with a recommendation to increase sunshine exposure and/or oral 25OHD supplementation. The result was made available to the medical clinic at routine follow up visits.

\section{Results}

\section{Socio-demographic characteristics}

Of the 168 patients screened, 142 patients were enrolled consecutively. Among the 26 ineligible patients, an alternative diagnosis was found in 12 patients and no reliable source of history was available for 12 others. For 2 patients, informed consent was not given. The response rate was $91 \%$ (142 of 156 patients with stroke). The overall median age (interquartile range (IQR) of patients was 69 (50-70) years; predominantly females, $54 \%$; residing in urban and peri-urban areas, $62 \%$; and had attended primary education, $61 \%$ (Table 1 ). The median duration of symptoms at enrolment was 5 days (IQR $3-7$ ), and most patients (90\% of 142) were enrolled within 10 days of onset of the symptoms. 
Table 1 Clinical characteristics associated with vitamin D deficiency among in-patients with acute stroke in Kampala Uganda- bivariate analysis

\begin{tabular}{|c|c|c|c|c|c|}
\hline Variable & $\begin{array}{l}\text { Total N (\% of total) or } \\
\text { median (IQR) }\end{array}$ & $\begin{array}{l}25 \mathrm{OHD}<20 \mathrm{ng} / \mathrm{ml} \mathrm{n} \text { (\% of row) } \\
\text { or median (IQR) }\end{array}$ & Crude odds ratio (OR) & $95 \%$ confidence interval & $P$ value \\
\hline \multicolumn{6}{|l|}{ Gender } \\
\hline Male & $69(49)$ & $12(17)$ & 1.00 & & \\
\hline Female & $73(51)$ & $9(12)$ & 0.67 & $0.27-1.70$ & 0.40 \\
\hline \multicolumn{6}{|l|}{ Hypertension history } \\
\hline No & $67(47)$ & $7(10)$ & 1.00 & & \\
\hline Yes & $75(53)$ & $14(19)$ & 1.97 & $0.74-5.21$ & 0.17 \\
\hline \multicolumn{6}{|l|}{ Diabetes mellitus } \\
\hline No & $117(82)$ & $18(15)$ & 1.00 & & \\
\hline Yes & $25(18)$ & $3(12)$ & 0.73 & $0.20-2.72$ & 0.64 \\
\hline \multicolumn{6}{|l|}{ HIV status } \\
\hline Negative & $130(92)$ & $19(15)$ & 1.00 & & \\
\hline Positive & $12(8)$ & $2(17)$ & 1.16 & $0.24-5.75$ & 0.85 \\
\hline \multicolumn{6}{|l|}{ Previous Stroke } \\
\hline No & $127(89)$ & $15(12)$ & 1.00 & & \\
\hline Yes & $15(11)$ & $6(40)$ & 4.98 & $1.55-15.96$ & 0.01 \\
\hline \multicolumn{6}{|l|}{ Typical dress } \\
\hline Gomesi $^{a}$ & $48(34)$ & $5(10)$ & 1.00 & & \\
\hline Short sleeve + trouser & $33(23)$ & $7(21)$ & 2.32 & $0.67-8.05$ & 0.19 \\
\hline Long sleeve + trouser & $28(20)$ & $4(14)$ & 1.43 & $0.35-5.84$ & 0.62 \\
\hline Other & $33(23)$ & $5(15)$ & 1.54 & $0.41-5.79$ & 0.53 \\
\hline \multicolumn{6}{|l|}{ Obesity } \\
\hline No & $81(57)$ & $13(16)$ & 1.00 & & \\
\hline Yes & $61(43)$ & $8(14)$ & 0.79 & $0.30-2.04$ & 0.63 \\
\hline \multicolumn{6}{|l|}{ Stroke type } \\
\hline Ischemic & $93(76)$ & $17(18)$ & 1.00 & & \\
\hline Hemorrhagic & $30(24)$ & $2(7)$ & 0.32 & $0.27-1.70$ & 0.14 \\
\hline bAge (years) & $69(50-70)$ & $70(62-79)$ & 1.03 & $1.00-1.06$ & 0.08 \\
\hline Daily time outdoors (h) & $6.75(3-10)$ & $3(1.5-7.5)$ & 0.85 & $0.75-0.96$ & 0.02 \\
\hline Exercise (days per week) & $1(0-7)$ & $1(0-7)$ & 0.95 & $0.82-1.10$ & 0.51 \\
\hline Systolic BP (mmHg) & 155 (139-171.5) & $150(141-170)$ & 1.00 & $0.98-1.01$ & 0.58 \\
\hline Diastolic BP (mmHg) & $90(77-101.5)$ & $90(80-103)$ & 1.00 & $0.98-1.01$ & 0.71 \\
\hline Corrected $\mathrm{Ca}^{++}(\mathrm{mmol} / \mathrm{l})$ & $2.32(2.22-2.39)$ & $2.31(2.23-2.36)$ & 0.21 & $0.01-5.38$ & 0.34 \\
\hline Random glucose (mmol/l) & $6.7(5.7-8.6)$ & $6.7(5.9-7.1)$ & 1.09 & $0.91-1.30$ & 0.33 \\
\hline Albumin (g/L) & $37.4(33.5-41.0)$ & $36.6(30.5-40.6)$ & 0.93 & $0.86-1.01$ & 0.07 \\
\hline Total cholesterol (mmol/L) & $4.53(3.59-5.45)$ & $4.58(3.18-5.4)$ & 0.89 & $0.63-1.24$ & 0.49 \\
\hline HDL cholesterol (mmol/L) & $1.18(0.94-1.43)$ & $0.58(1.31-1.15)$ & 0.25 & $0.07-0.92$ & 0.04 \\
\hline
\end{tabular}

a Uganda national women's dress covering lower limbs and torso leaving arms bare

${ }^{b}$ Data from this row onward are continuous; association with the categorical outcome of vitamin D deficiency is displayed as odds ratios

\section{Clinical and laboratory findings}

A previous history of hypertension was obtained in 75 patients (53\% of 142). Diabetes mellitus was found in 25 patients (19\% of 142); 19 patients by history of DM, and an additional 6 patients based on a high random blood glucose measurement. Other co-existing co-morbidities were HIV infection, non-atrial fibrillation cardiac disease, atrial fibrillation and chronic kidney disease in 9, 6, 
4 and $3 \%$ of the patients respectively. One patient had sickle cell disease and one patient had tuberculosis.

Antiretroviral therapy was used by 5 of the $12 \mathrm{HIV}$ positive subjects, with majority (60 \%) using an efavirenz based regimen. The use of multivitamin supplements was not found in any patient.

Prior history of stroke was reported in 15 (11\%) patients. A brain CT scan was done in 123 patients $(87 \%$ of 142). However, 19 patients (13\% of 142) did not get brain CT scans due to financial constraints. Ischemic stroke was the most prevalent type of stroke, noted in 93 (76\%) patients.

The median HDL Cholesterol (HDLC) and total cholesterol (TC) in $\mathrm{mmol} / \mathrm{l}$ was 1.18 (0.94-1.43), and 4.35 (3.59-5.45) respectively. The median calcium concentration, corrected for albumin levels in was $2.32 \mathrm{mmol} / \mathrm{l}$ $(1.99-2.59)$

\section{OHD status and related factors}

The prevalence of vitamin D deficiency was $15 \%$ (21 out of 142 patients). This included 5 patients ( $4 \%$ of 142 ) who were categorized as having severe vitamin $\mathrm{D}$ deficiency. Vitamin D insufficiency (25OHD 20- $<30 \mathrm{ng} / \mathrm{ml}$ ) was found in 29 patients ( $20 \%$ of 142). Overall two thirds (65\% of 142) of the study patients had adequate vitamin D levels. The median (IQR) concentration of $25 \mathrm{OHD}$ was $36.4 \mathrm{ng} / \mathrm{ml}(22.0-45.7)$.

The burden of vitamin D deficiency was greater among patients with ischemic stroke than in patients with hemorrhagic strokes, although not statistically significant (18\% vs. $7 \%, p=0.33)$. An age adjusted prevalence of vitamin $\mathrm{D}$ deficiency by stroke type (computed using the age distribution of the entire sample) was similar to the crude prevalence, at $19 \%$ versus $9 \%$ in patients with ischemic and hemorrhagic strokes respectively.

Among subjects with vitamin D deficiency, there were more patients with a history of hypertension $(n=14$, $67 \%$ of 21$)$ and more male patients $(n=12,57 \%$ of 21$)$. Vitamin D deficiency was found in 6 (29\% of 15) patients with a previous history of stroke, and $2(17 \%$ of 12) patients with HIV. None of the patients on antiretroviral therapy had vitamin $\mathrm{D}$ deficiency.

No significant linear relationship was observed between 25OHD and systolic blood pressure $(r=-0.04, p=0.62)$, 25OHD and diastolic blood pressure $(r=-0.05, p=0.58)$, or 25OHD and random blood glucose $(r=-0.03, p=0.69)$.

At bivariate analysis, increasing age, prior history of a stroke, hours spent outdoors, increasing albumin and HDLC concentrations had $p$ values $\leq 0.1$ and were entered into the multivariate analysis model, Gender, diabetes mellitus, obesity, and a history of hypertension were also included as important confounders (Table 2). Lower HDLC concentrations (OR 0.09, $p=0.02$ ) and shorter duration outdoors (OR $0.83, p=0.02$ ) were independently
Table 2 Clinical characteristics associated with vitamin D deficiency among in-patients with acute stroke in Kampala Uganda-multivariate analysis

\begin{tabular}{|c|c|c|c|c|}
\hline Variable & $\begin{array}{l}\text { Crude } \\
\text { odds ratio }\end{array}$ & $\begin{array}{l}\text { Adjusted } \\
\text { odds ratio }\end{array}$ & $95 \% \mathrm{Cl}$ & $P$ value \\
\hline \multicolumn{5}{|l|}{ Gender } \\
\hline Male & 1.00 & & & \\
\hline Female & 0.67 & 1.18 & $0.38-3.67$ & 0.77 \\
\hline \multicolumn{5}{|l|}{ Diabetes } \\
\hline No & 1.00 & & & \\
\hline Yes & 0.73 & 0.29 & $0.41-2.10$ & 0.22 \\
\hline \multicolumn{5}{|l|}{ Obesity } \\
\hline No & 1.00 & & & \\
\hline Yes & 0.79 & 0.71 & $0.21-2.37$ & 0.57 \\
\hline \multicolumn{5}{|l|}{ Hypertension history } \\
\hline No & 1.00 & & & \\
\hline Yes & 1.97 & 2.00 & $0.62-6.43$ & 0.25 \\
\hline \multicolumn{5}{|l|}{ Previous stroke } \\
\hline No & 1.00 & & & \\
\hline Yes & 4.98 & 3.80 & $0.94-15.32$ & 0.06 \\
\hline${ }^{\mathrm{a} A g e(y e a r s)}$ & 1.03 & 1.04 & $1.00-1.08$ & 0.05 \\
\hline Daily time outdoors (h) & 0.85 & 0.83 & $0.71-0.97$ & 0.02 \\
\hline Albumin $(\mathrm{g} / \mathrm{L})$ & 0.93 & 1.01 & $0.90-1.13$ & 0.92 \\
\hline HDL cholesterol & 0.25 & 0.09 & $0.01-0.68$ & 0.02 \\
\hline
\end{tabular}

${ }^{\mathrm{a}}$ From this row onwards, continuous predictor variables are displayed, with odds ratio of vitamin $\mathrm{D}$ deficiency per unit rise in the predictor variable

associated with vitamin D deficiency among stroke patients in the multivariate analysis model. A previous history of stroke (OR 3.8, $p=0.06$ ) and increasing age (OR 1.04, $p=0.05$ ) were not significantly associated with vitamin $\mathrm{D}$ deficiency.

\section{Discussion}

In this study, we found a relatively low prevalence of vitamin $\mathrm{D}$ deficiency among adult in-patients with acute stroke at a referral hospital in East Africa. The significant factors associated with vitamin D deficiency in this group were a lower reported duration of sunshine exposure and lower HDL cholesterol. To our knowledge, this study is the first in SSA to describe the burden of vitamin D deficiency among patients with acute stroke or other cardiovascular diseases.

Comparing studies that report vitamin D deficiency requires attention to the $25 \mathrm{OHD}$ cut-off values used to define it. Different cut-off values have been used among studies in East African and elsewhere, ranging from $<10 \mathrm{ng} / \mathrm{ml}$ to $<30 \mathrm{ng} / \mathrm{ml}[6,31,33,34]$. The various cut-off values reflect the changing understanding of the roles of 25OHD beyond only bone 
health, to include roles in immunology and infections, mental illness, cardiovascular prevention, cancer, and other health outcomes [1, 12, 24, 25, 33, 35]. A cutoff value of $<20 \mathrm{ng} / \mathrm{ml}$ is widely accepted through consensus among experts [1, 31, 33]. It is also frequently reported even by studies that define vitamin $\mathrm{D}$ deficiency otherwise. For purposes of this study, we took care to compare 25OHD $<20 \mathrm{ng}$ in reference to vitamin $\mathrm{D}$ deficiency.

Our findings on the prevalence of vitamin D deficiency are similar to those in other studies in the region of SSA: in Uganda this prevalence has been 9-20\% among normal study subjects $[5,6]$. Among in-patients on a medical ward in Malawi a prevalence of $14 \%$ was found [36]. However, an examination of data from other studies reveals wide contrasts in the SSA region: among traditionally living populations in East Africa have reported a high mean $25 \mathrm{OHD}$ value with virtually no vitamin D deficiency [37]. In contrast, studies in central Ethiopia and Northern Nigeria have reported high prevalence of suboptimal 25OHD levels [7, 8].

We found that lower sunshine exposure was associated with vitamin D deficiency in this study. This is expected since ultraviolet $B$ (UVB) radiation in sunshine drives cutaneous vitamin $D$ synthesis [1]. Estimating exposure to sunshine using a questionnaire to establish typical intervals spent outdoors is a method that has been used with some success, but it is limited by recall bias and variation in behaviour during time spent outdoors [38].

The association between older age and vitamin D deficiency was not statistically significant, probably due to the limited sample size and study power. An association between rising age and vitamin D deficiency was the expected; it is biologically plausible due to a decline in the efficiency of cutaneous vitamin D synthesis with increasing age $[1,39]$. Paradoxically, another recent study in an urban population in East Africa reported lower levels of 25OHD among younger adults compared to older adults [40]. This may reflect changing lifestyles and decreased sunshine exposure among younger urban populations.

Other factors including, religion, cultural practices, diet, co-morbidities and low awareness may contribute to disparities in the prevalence of vitamin D deficiency seen in the tropics [2, 3]. Genotype of vitamin D binding protein and the type of 25OHD (25OHD2 or 25OHD3) are linked to changes in the serum half life of vitamin D deficiency and the likelihood of vitamin D deficiency [30].

Outside the tropics, studies among patients with acute stroke/cardiovascular events generally report greater prevalence of vitamin D deficiency than we found. In Europe and South East Asia, studies also report a greater burden of vitamin D deficiency among stroke patients compared to control subjects in the same environment $[16,41]$. Our study design did not include similar control subjects, limiting our ability to make meaningful comparisons.

Among patients with stroke vitamin D deficiency is reported to predict greater severity and adverse outcomes including recurrent strokes and death [26, 42]. Vitamin D deficiency is also linked with a greater likelihood of falls, poor muscle and bone health, and possibly increased fracture risk among stroke survivors [16, 43]. On the other hand stroke predisposes to vitamin D deficiency, possibly through reduced dietary intake, inability to feed independently, and decreased mobility and sunshine exposure $[16,43]$. As expected, we found a higher burden of vitamin $\mathrm{D}$ deficiency among the subjects who had suffered at least one previous stroke. Nonetheless, no significant association was found after adjusting for other factors perhaps due to low study power. However, we did not examine the relation of 25OHD status with stroke severity or mortality in our setting. Routine strategies to prevent vitamin D deficiency among stroke survivors in our setting are required.

While vitamin D deficiency is an independent predictor for stroke and other cardiovascular events, it is also associated with traditional risk factors like hypertension, insulin resistance/diabetes, obesity, abnormal lipid profile $[24,44]$. Our study indeed showed that the likelihood of vitamin D deficiency increased with a declining HDL level. Studies among immigrants from SSA in Australia have documented vitamin D deficiency clustered with obesity, insulin resistance and dyslipidemia [23], and go ahead to propose a role for vitamin D replacement in cardiovascular prevention [27]. In addition, vitamin $\mathrm{D}$ deficiency is related to novel cardiovascular risk factors such as C-Reactive Protein and proinflammatory cytokines [24, 44]. We were not able to demonstrate an association between vitamin D deficiency and hypertension-the single most powerful cardiovascular risk factor in SSA today [18].

Importantly the diagnosis of hypertension and diabetes in our study were influenced by low awareness of either condition, physiological responses of blood pressure and blood sugar to acute stroke [45], and absence of supporting tests like the HBA1c assay.

In this region with rising incidence of cardiovascular risk factors and diseases, vitamin D deficiency appears to contribute a minor fraction to the burden at present. The burden of vitamin $\mathrm{D}$ deficiency in relation to stroke/cardiovascular diseases may increase with greater longevity, and behavioural change including lower exposure to sunshine.

\section{Limitations}

Due to logistical constraints, low numbers were recruited in our study, thus limiting the power of the study. The 
diagnosis of acute stroke was made based on clinical history and physical exam findings within 2 weeks of onset, with or without corroborating imaging studies. While this could limit the accuracy of stroke diagnosis, similar approaches have been used in other studies on stroke and vitamin D deficiency. We included all stroke subtypes in this study. Previous studies have not always made a distinction between hemorrhagic and ischemic strokes. Mechanisms by which vitamin D deficiency is related to stroke relate more to atherosclerosis and ischemic stroke.

Different 25OHD assays were used in the studies we have compared, but the new Roche vitamin D total assay that we used has demonstrated strong correlation with high performance liquid chromatography/mass spectrometry and established radioimmunoassay techniques [46]. Due to our study design, we are unable to show a temporal relationship between vitamin $\mathrm{D}$ deficiency and stroke events. However, studies elsewhere have frequently found that vitamin D deficiency is associated with stroke, and may indeed predict stroke [19]. Ongoing studies elsewhere are investigating the role of $25 \mathrm{OHD}$ replacement on non communicable diseases including cardiovascular disease, cerebrovascular disease and cancer [28].

\section{Conclusions}

There was a relatively low burden of vitamin D deficiency among patients with acute stroke in Uganda. Vitamin D deficiency plays a minor role in stroke risk in Uganda at present. With increasing longevity and indoor lifestyles vitamin D deficiency may assume a greater role in stroke and other cardiovascular diseases in tropical sub Saharan Africa. Future studies may be necessary to evaluate the relationship of vitamin $\mathrm{D}$ deficiency to stroke outcomes and the mechanisms thereof.

\section{Additional file}

Additional file 1: Study data Collection form. (PDF $83 \mathrm{~kb}$ )

\section{Competing interests}

Authors declare no competing interests. The opinions expressed by the authors contributing to this journal do not necessarily reflect the opinions of the authors' affiliated institutions or the official views of the National Institutes of Health.

\section{Authors' contributions}

Concept development and study design: DSK, DK, RB, BK, BKK, and EK. Data Collection: DSK. Drafting the first manuscript: DSK, DK. Data analysis: DSK, EM. Manuscript writing, first draft and critical review of manuscript: All authors. Study oversight/supervision EM, RB, and EK. All authors read and approved the final draft of manuscript before submission.

\section{Acknowledgements}

The authors thank all the patients/family caregivers who participated in this study, and Dr. Martina Nanteza who performed the statistical analysis. The Medical Educational Partnership Initiative-Cardiovascular Diseases Linked award (MEPI-CVD)-under Prof Moses Kamya and Prof Nelson Sewankambo-was instrumental in obtaining support for this publication.
Research reported in this publication was supported in part by the Fogarty International Center, the National Heart Lung and Blood Institute, and the Common Fund of the National Institutes of Health under Award Number R24TW008861.

\section{Author details}

${ }^{1}$ Department of Medicine, College of Health Sciences, Makerere University, Kampala, Uganda. ${ }^{2}$ Department of Medicine, Uganda Martyrs Hospital, Lubaga, Kampala, Uganda. ${ }^{3}$ Department of Medicine, Case Western Reserve University, Cleveland, OH, USA. ${ }^{4}$ Uganda Heart Institute, Mulago Hospital, Kampala, Uganda. ${ }^{5}$ Uganda Virus Research Institute, Entebbe, Uganda.

Received: 22 April 2014 Accepted: 1 October 2015

Published online: 05 October 2015

\section{References}

1. Holick MF. Vitamin D Deficiency. N Engl J Med. 2007;357:266-81.

2. Arabi A, El Rassi R, El-Hajj Fuleihan G. Hypovitaminosis D in developing countries - prevalence, risk factors and outcomes. Nat Rev Endocrinol. 2010;6:550-61.

3. Prentice A, Schoenmakers I, Jones K, Jarjou LA, Goldberg G. Vitamin D Deficiency and Its Health Consequences in Africa. Clin Rev Bone Miner Metab. 2009;7:94-106.

4. Schoenmakers I, Goldberg GR, Prentice A. Abundant sunshine and vitamin D deficiency. Br J Nutr. 2008;99:1171

5. Conesa-Botella A, Goovaerts O, Massinga-Loembé M, Worodria W, Mazakpwe $D$, Luzinda $K$, et al. Low prevalence of vitamin D deficiency in Ugandan HIV-infected patients with and without tuberculosis. Int J Tuberc Lung Dis. 2012;16:1517-21.

6. Nansera D, Graziano FM, Friedman DJ, Bobbs MK, Jones AN, Hansen KE. Vitamin D and calcium levels in Ugandan adults with human immunodeficiency virus and tuberculosis. Int J Tuberc Lung Dis. 2011;15:1522-8.

7. Glew RH, Crossey MJ, Polanams J, Okolie HI, VanderJagt DJ. Vitamin D Status of Seminomadic Fulani Men and Women. J Natl Med Assoc. 2012;102:485-90.

8. Wakayo T, Belachew T, Vatanparast H, Whiting SJ. Vitamin D Deficiency and Its Predictors in a Country with Thirteen Months of Sunshine: The Case of School Children in Central Ethiopia. PLoS One. 2015;10:e0120963.

9. Ruwanpathirana T, Reid CM, Owen AJ, Fong DPS, Gowda U, Renzaho AMN. Assessment of vitamin $\mathrm{D}$ and its association with cardiovascular disease risk factors in an adult migrant population: an audit of patient records at a Community Health Centre in Kensington, Melbourne. Australia BMC Cardiovasc Disord. 2014;14:157.

10. Pirrone A, Capetola T, Riggs E, Renzaho A. Vitamin D deficiency awareness among African migrant women residing in high-rise public housing in Melbourne, Australia: a qualitative study. Asia Pac J Clin Nutr. 2013;22:292-9.

11. Kibirige D, Mutebi E, Ssekitoleko R, Worodria W, Mayanja-Kizza H. Vitamin D deficiency among adult patients with tuberculosis: a cross sectional study from a national referral hospital in Uganda. BMC Res Notes. 2013;6:293.

12. Cusick SE, Opoka RO, Lund TC, John CC, Polgreen LE. Vitamin D insufficiency is common in ugandan children and is associated with severe malaria. PLoS One. 2014;9:e113185.

13. Wang TJ, Pencina MJ, Booth $S L$, Jacques PF, Ingelsson E, Lanier K, et al. Vitamin D deficiency and risk of cardiovascular disease. Circulation. 2008;117:503-11.

14. Moy F-M, Bulgiba A. High prevalence of vitamin D insufficiency and its association with obesity and metabolic syndrome among Malay adults in Kuala Lumpur. Malaysia BMC Public Health. 2011;11:735.

15. Mitri J, Muraru MD, Pittas AG. Vitamin D and type 2 diabetes: a systematic review. Eur J Clin Nutr. 2011;65:1005-15.

16. Poole KES. Reduced vitamin D in acute stroke. Stroke. 2005;37:243-5.

17. Lopez ADAD, Mathers CDCD, Ezzati MM, Jamison DTDT, Murray CJCJL, editors. Global Burden of Disease and Risk Factors. Washington (DC): World Bank; 2006.

18. Mensah GA. Epidemiology of stroke and high blood pressure in Africa. Heart. 2008;94:697.

19. Pilz S, Dobnig H, Fischer JE, Wellnitz B, Seelhorst U, Boehm BO, et al. Low vitamin $\mathrm{D}$ levels predict stroke in patients referred to coronary angiography. Stroke. 2008:39:2611-3.

20. Cheng S, Massaro JM, Fox CS, Larson MG, Keyes MJ, McCabe EL, et al. Adiposity, cardiometabolic risk, and vitamin D status: the framingham heart study. Diabetes. 2009;59:242-8. 
21. Forman JP, Curhan GC, Taylor EN. Plasma 25-hydroxyvitamin D levels and risk of incident hypertension among young women. Hypertension. 2008;52:828-32.

22. Maki KC, Rubin MR, Wong LG, McManus JF, Jensen CD, Marshall JW, et al. Serum 25-hydroxyvitamin D is independently associated with high-density lipoprotein cholesterol and the metabolic syndrome in men and women. J Clin Lipidol. 2009;3:289-96.

23. Renzaho AM, Nowson C, Kaur A, Halliday JA, Fong D, Desilva J. Prevalence of vitamin D insufficiency and risk factors for type 2 diabetes and cardiovascular disease among African migrant and refugee adults in Melbourne: a pilot study. Asia Pac J Clin Nutr. 2011;20:397-403.

24. Artaza JN, Mehrotra R, Norris KC. Vitamin D and the cardiovascular system. Clin J Am Soc Nephrol. 2009;4:1515-22.

25. Cannell JJ, Grant WB, Holick MF. Vitamin D and inflammation. Dermatoendocrinol. 2014;6:e983401.

26. Daubail B, Jacquin A, Guilland J-C, Hervieu M, Osseby G-V, Rouaud O, et al. Serum 25-hydroxyvitamin D predicts severity and prognosis in stroke patients. Eur J Neurol. 2013;20:57-61.

27. Ruwanpathirana T, Owen A, Renzaho AM, Zomer E, Gambhir M, Reid CM Can oral vitamin D prevent the cardiovascular diseases among migrants in Australia? Provider perspective using Markov modelling. Clin Exp Pharmacol Physiol. 2015;42:596-601

28. Manson JE, Bassuk SS, Lee I-M, Cook NR, Albert MA, Gordon D, et al. The VITamin D and OmegA-3 TriaL (VITAL): Rationale and design of a large randomized controlled trial of vitamin $D$ and marine omega-3 fatty acid supplements for the primary prevention of cancer and cardiovascular disease. Contemp Clin Trials. 2012;33:159-71.

29. Chin $\mathrm{JH}$. Stroke in Sub-Saharan Africa: an urgent call for prevention. Neurology. 2012;78:1007-8.

30. Braithwaite VS, Jones KS, Schoenmakers I, Silver M, Prentice A, Hennig BJ. Vitamin D binding protein genotype is associated with plasma $25 \mathrm{OHD}$ concentration in West African children. Bone. 2015;74:166-70.

31. Holick MF, Binkley NC, Bischoff-Ferrari HA, Gordon CM, Hanley DA, Heaney RP, et al. Evaluation, treatment, and prevention of vitamin D deficiency: an endocrine society clinical practice guideline. J Clin Endocrinol Metab. 2011;96:1911-30

32. Alberti $K G$, Zimmet $P$, Shaw J. A consensus statement from the international diabetes federation. Diabet Med. 2006;23:469-80.

33. Gallagher JC, Sai AJ. Vitamin D insufficiency, deficiency, and bone health. J Clin Endocrinol Metab. 2010;95:2630-3.

34. Stroud ML, Stilgoe S, Stott VE, Alhabian O, Salman K. Vitamin D - a review. Aust Fam Physician. 2008;37:1002-5.

35. Mehta S, Giovannucci E, Mugusi FM, Spiegelman D, Aboud S, Hertzmark E, et al. Vitamin D status of HIV-infected women and its association with HIV disease progression, anemia, and mortality. PLoS One. 2010;5:e8770.

36. Mastala Y, Nyangulu P, Banda RV, Mhemedi B, White SA, Allain TJ. Vitamin $D$ deficiency in medical patients at a central hospital in Malawi: a comparison with TB patients from a previous study. PLoS One. 2013;8:e59017.

37. Luxwolda MF, Kuipers RS, Kema IP, Janneke Dijck-Brouwer DA, Muskiet FAJ. Traditionally living populations in East Africa have a mean serum 25-hydroxyvitamin D concentration of $115 \mathrm{nmol} / \mathrm{l}$. Br J Nutr. 2012;108:1557-61.

38. McCarty CA. Sunlight exposure assessment: can we accurately assess vitamin D exposure from sunlight questionnaires? Am J Clin Nutr. 2008;87:1097S-101.

39. MacLaughlin J, Holick MF. Aging decreases the capacity of human skin to produce vitamin D3. J Clin Invest. 1985;76:1536-8.

40. Theuri G, Kiplamai F. Association between vitamin D levels and central adiposity in an eastern Africa outpatient clinical population. Dermatoendocrinol. 2013,5:218-21.

41. $\mathrm{Ng} J$. Prevalence of vitamin D deficiency among ethnic asian ischaemic stroke patients and relationship to underlying stroke aetiology. 2012

42. Balden R, Selvamani A, Sohrabji F. Vitamin D deficiency exacerbates experimental stroke injury and dysregulates ischemia-induced inflammation in adult rats. Endocrinology. 2012;153:2420-35.

43. Makariou SE, Michel P, Tzoufi MS, Challa A, Milionis HJ. Vitamin D and stroke: promise for prevention and better outcome. Curr Vasc Pharmacol. 2012;12:117.
44. Lavie CJ, Lee JH, Milani RV. Vitamin D and cardiovascular disease. J Am Coll Cardiol. 2011;58:1547-56

45. Wong AA, Read SJ. Early changes in physiological variables after stroke. Ann Indian Acad Neurol. 2008;11:207-20.

46. Emmen JMA, Wielders JPM, Boer A-K, van den Ouweland JMW, Vader HL. The new Roche vitamin D total assay: fit for its purpose? Clin Chem Lab Med. 2012;0:1-4

\section{Submit your next manuscript to BioMed Central and take full advantage of:}

- Convenient online submission

- Thorough peer review

- No space constraints or color figure charges

- Immediate publication on acceptance

- Inclusion in PubMed, CAS, Scopus and Google Scholar

- Research which is freely available for redistribution

Submit your manuscript at www.biomedcentral.com/submit 